\title{
'Speed collaborations' and the quality versus quantity debate in ecology and evolution publications
}

\author{
Michael R. Donaldson \\ Michael R. Donaldson (michael.r.donaldson@gmail.com) Department of Natural Resources and Environmental \\ Sciences, University of Illinois at Urbana-Champaign, 1102 S. Goodwin Ave, Urbana, IL 61801 USA
}

\section{Background}

The argument over whether quality or quantity is more valuable to the advancement of ecology and evolutionary science continues to rage (Fischer et al. 2012, Loyola et al. 2012). With research quantity (i.e., number of papers published) leading to higher citation rates and increased funding (Lortie et al. 2012), some researchers have made the call for their colleagues to refocus efforts in order to emphasize the roles of creativity and communication to inspire quality research (e.g., Fischer et al. 2012). The quality and quantity spectrum does not need to represent a trade-off; both attributes are important to the advancement of science and should not be considered mutually exclusive. Unfortunately, finding equilibrium between quality and quantity is a difficult balancing act in the electronic era.

How then, can we ensure both quality and quantity in scientific research? The answer may lie in innovative collaborations. Collaboration among researchers is immensely powerful and, arguably perhaps, becoming a necessary means of advancing knowledge. To remain influential and to keep pace in a fast moving world, researchers must find new ways to spark scientific creativity, and must do so in a timely manner. One novel approach to tackle this is short duration, focused, multi-disciplinary collaborations, herein referred to as 'speed collaborations' (Favaro et al. 2013). Here, I argue that expanding on this new view of rapid, collaborative research could bridge the gap between quality and quantity research by (i) generating new ideas, (ii) enhancing networks, and (iii) facilitating communication over a compressed time-scale. i. Speed collaborations to spark ideas and ignite creative scientific thinking

While it has been argued that high pressure environments can hinder creativity (Shalley and Gilson 2004), Favaro et al. (2013) present a case study of a highly effective means of creative idea generation under pressure via the Research Derby. When pushed outside of their typical research areas and placed under a strict timeline of only hours, graduate students were able to generate and develop novel and exciting research ideas of broad interest and scope. The Research Derby exemplifies how a series of high quality research ideas can be produced in a timely manner. Similar examples of speed collaborations have taken place in recent years by Sutherland and colleagues as part of their 100 questions exercise (Sutherland et al. 2009) and of their annual horizon scan exercises (e.g., Sutherland et al. 2013). These highly cited and influential works are the results of assembling expert panels of researchers from a range of disciplines to come together for rapid (i.e. hours to days), focused collaborative face-to-face meetings to discuss topics and generate ideas. The pressure cooker mentality associated with these types of speed collaborations may be intimidating, but may ultimately help to quickly generate ideas and publications in an effort to produce research in both quality and quantity.

\section{ii. Speed collaborations to build multi-disciplinary research networks}

Speed collaborations offer opportunities for developing and strengthening networks. In terms of network 
development, Favaro and Braun (2013) provide evidence that there is great value in bringing graduate students together that might otherwise not work together (i.e., from different research labs) since it helps individuals build their own personal networks and also provides experience in working with others outside their own research group. Speed collaborations may help to strengthen existing networks by marrying a range of participants from various disciplines and sub-disciplines that each bring their own unique expertise and perspectives to the table. For example, the Sutherland et al. $(2009,2013)$ speed collaborations highlight how a range of experts from diverse disciplines can work together towards common goals. The power in this approach is that networks that are established and solidified during speed collaborations may lead to future collaborations over many years (e.g., Sutherland's horizon scans) and produce quality and quantity work over the long-term. The challenge for speed collaborations is in the establishment of networks in the first place (i.e., who should be included in speed collaborations and how many levels of organization should be targeted?). The answer to that question will depend on the context and goals associated with the speed collaboration and, ultimately, on the intended audience the researchers are attempting to reach (e.g., policy-makers; Sutherland et al. 2011).

\section{iii. Speed collaborations to communicate ideas to diverse audiences}

Provided that speed collaborations remain multidisciplinary, they offer a unique opportunity to communicate new ideas to a range of audiences. As Favaro et al. (2013) described, the ideas generated during their Research Derby were of broad appeal and general interest to a range of conservation biologists, ecologists and evolutionary biologists. Perhaps the best example of this is the Phillis et al. (2012) paper that resulted from the Research Derby on the topic of how scientists, policymakers and the public engage in conservation science, which is big picture in scope, applicable to a range of research interests, and consequently published in a general interest conservation science journal, Conservation Letters. Likewise, the Sutherland et al. (e.g., 2013) horizon scan exercises continue to generate a great deal of interest from other researchers (i.e., high citation rate) and the media, which in turn could garner further attention from funding agencies, policy-makers, and the public. With speed collaborations having the power to generate big ideas, there is great potential for these influential ideas to reach wide-ranging audiences. With the emphasis on speed, idea generation and communication can occur rapidly, which greatly facilitates the rapid dissemination of timely ideas (e.g., Sutherland et al. 2013) and could contribute to both quality and quantity in research.
The quality versus quantity debate will undoubtedly continue to rage, but striving towards consensus between the two camps by emphasizing new kinds of collaborations to bridge the gap will be important for moving forward. Speed collaborations have the potential to yield influential (Donaldson and Cooke in press), timely, and novel contributions to ecological and evolutionary science. In order to keep pace with a rapidly moving world, speed collaborations represent a new means of generating ideas, facilitating networking, and enhancing communication to move science forward in the electronic era.

\section{References}

Donaldson, M.R. and S.J. Cooke. In press. Scientific publication: Moving beyond quality and quantity toward influence. Bioscience.

Favaro, B., Braun, D.C. and Earth2Ocean Research Derby. 2013. The 'Research Derby': A pressure cooker for creative and collaborative science. Ideas in Ecology and Evolution 6:40-46. CrossRef

Fischer, J., Ritchie, E.G. and J. Hanspach. 2012. Academia's obsession with quantity. Trends in Ecology and Evolution 27:473-474. CrossRef

Lortie, C.J., Aarssen, L.W., Parker, J.N. and S. Allesina. 2012. Good news for the people who love bad news: an analysis of the funding of the top $1 \%$ most highly cited ecologists. Oikos 121: 1005-1008. CrossRef

Loyola, R.D., Diniz-Filho, J.A.F. and L.M. Bini. 2012. Obsession with quantity: a view from the south. Trends in Ecology and Evolution 27: 585. CrossRef

Phillis, C.C., O’Regan, S.M., Green, S.J., Bruce, J.E.B., Anderson, S.C., Linton, J.N., et al. 2012. Multiple pathways to conservation success. Conservation Letters 6:98-106. CrossRef

Shalley, C.E., and L.L. Gilson. 2004. What leaders need to know: a review of social and contextual factors that can foster or hinder creativity. The Leadership Quarterly 15:33-53. CrossRef

Sutherland, W.J., Adams, W.M., Aronson, R.B., Aveling, R., Blackburn, T.M., Broad, S. et al. 2009. One hundred questions of importance to the conservation of global biological diversity. Conservation Biology 23:557-567. CrossRef

Sutherland W.J., Fleishman, E., Mascia, M.B., Pretty, P. and M.A. Rudd. 2011. Methods for collaboratively identifying research priorities and emerging issues in science and policy. Methods in Ecology and Evolution 2:238-247. CrossRef

Sutherland, W.J., Bardsley, S., Clout, M., Depledge, M.H., Dicks, L.V., Fellman, L., et al. 2013. A horizon scan of global conservation issues for 2013. Trends in Ecology and Evolution 28: 16-22. CrossRef 